\title{
Prescient Beamforming in Multi-User Interweave Cognitive Radio Networks
}

\author{
(Invited Paper) \\ Amitav Mukherjee and A. Lee Swindlehurst \\ Electrical Engineering and Computer Science \\ University of California Irvine \\ \{a.mukherjee; swindle\}@uci.edu
}

\begin{abstract}
This work investigates a fundamentally novel interweave cognitive radio network where the primary transmitter takes a proactive approach towards improving the accuracy of the spectrum sensing outcomes at the secondary users (SUs). For the single-primary-receiver scenario considered here, the multiantenna primary user constructs its transmit beamforming vector so as to increase the detection probability at the SUs while meeting a desired Quality-of-Service (QoS) target on its own link, by exploiting partial channel state information of the SUs. The objective of such a proactive approach, which we refer to as prescient precoding, is to minimize the probability of interference from SUs at the primary receiver due to imperfect spectrum sensing in fading channels. Numerical results are presented to verify the advantages of the proposed prescient transmission techniques for non-cooperative spectrum sensing scenarios.
\end{abstract}

\section{INTRODUCTION}

Cognitive radios are emerging as promising solutions to enable better utilization of the radio spectrum, especially in bands that are currently under-utilized. The canonical model of a cognitive radio (CR) is one that employs "interweave" cognition [1]. Under the interweave paradigm, cognitive radios seek to opportunistically occupy a channel (frequency band) only when it is not occupied by a primary transmitter (PT) licensed to use that band. In the absence of pre-defined control channels or coordinated medium access between the primary and unlicensed users, the CRs must periodically sense the spectrum for the presence of PTs [1], [2] and cease transmission upon detection. Inevitably, imperfect CR spectrum sensing due to channel fading and other impairments will lead to unintentional interference at the primary receivers (PRs).

The use of multiple antennas in CRs has been suggested for improved spectrum sensing capabilities in interweave systems by means of receive diversity [2]. MIMO CRs have also been investigated in the context of "underlay" cognitive radio networks, where CRs and PTs coexist in the same spectrum and spectrum sensing is not required. In this case, multiple transmit antennas are used in the secondary network for beamforming and to minimize the interference to the PRs, with complete or partial channel state information (CSI) at the SU transmitter [3].

This work was supported by the National Science Foundation under gran CCF-0916073.
In all prior work on interweave networks, the primary transmitters are assumed to be legacy users that are oblivious to the presence of the secondary users. However, the problem considered in this work is fundamentally different in that the PTs construct their transmit beamformers with the objective of increasing the detection probability at the secondary users (SUs) while meeting a desired QoS target on its own link, given partial CSI of the PRs and SUs. Therefore, the primary transmitter adopts a proactive approach, which we refer to as prescient beamforming, to minimize the probability of interference from SUs at the primary receiver due to imperfect spectrum sensing. Furthermore, we analyze an interweave network where the primary transmitter is equipped with multiple antennas, while the primary receiver and secondary transmitters/receivers have a single antenna each. The potential benefits of multi-antenna PTs and PRs have received surprisingly little attention in the literature on both interweave and underlay $\mathrm{CR}$ systems.

The remainder of this paper is organized as follows. Section II introduces the primary transmission and SU spectrum sensing strategies, and examines the impact of SU misdetections on the primary receiver. Section III presents the details of two prescient beamforming algorithms for a single primary link and multiple SUs. Selected numerical examples are shown in Section IV, and we conclude in Section V.

\section{MAthematical Model}

\section{A. Signal and Network Model}

Consider a network with a single $N$-antenna PT, its intended PR with a single antenna, and $K$ secondary users (SUs, also synonymous with secondary transmitters), as depicted in Fig. 1. Let the primary terminal be denoted as user 0, and assume that the PT employs linear transmit beamforming. When active, the PT transmit signal at time index $t$ is given by

$$
\mathbf{x}(t)=\mathbf{w} s_{0}(t)
$$

where $\mathbf{w} \in \mathbb{C}^{N \times 1}$ is the transmit beamforming weight vector and $s_{0}(t)$ is the complex scalar zero-mean information symbol transmitted at $t$, with power $E\left\{\left|s_{0}(t)\right|^{2}\right\}=\sigma_{s}^{2}$. A constraint is assumed to be imposed on the average transmit power of 


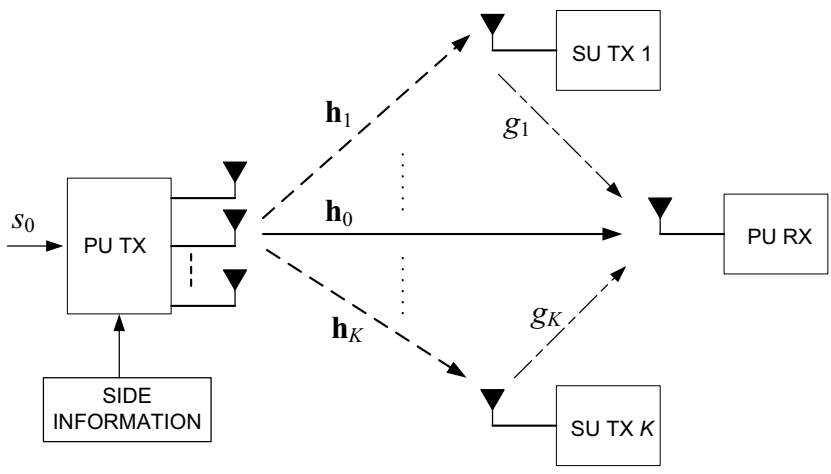

Fig. 1. Cognitive radio network with a multi-antenna primary transmitter a single primary receiver, and $K$ secondary transmitters. The secondary receivers are not shown for clarity.

the PT:

$$
E\left\{\operatorname{Tr}\left(\mathbf{x}(t) \mathbf{x}(t)^{H}\right)\right\} \leq P \quad \forall t .
$$

When the PT is active, the signal received by the $k^{t h} \mathrm{SU}$ if in sensing mode is

$$
y_{k}(t)=\mathbf{h}_{k}(t) \mathbf{x}(t)+z_{k}(t),
$$

where $z_{k}(t)$ is additive complex Gaussian noise with zero mean and variance $\sigma_{k}^{2}$.

We will assume that partial CSI is available to the PT, which is defined to mean that the PT has knowledge of the instantaneous realizations of the PT-PR $\left(\mathbf{h}_{0}\right)$ and PTSU $\left(\left\{\mathbf{h}_{k}\right\}_{k=1}^{K}\right)$ channels but only the statistics of the SU-PR channels $\left(\left\{g_{k}\right\}_{k=1}^{K}\right)$.

\section{B. SU Spectrum Sensing}

We assume that the SUs perform non-cooperative spectrum sensing and are not all simultaneously in spectrum-sensing mode. We capture this assumption by assigning a probability $\beta$ to the event that an arbitrary SU is in spectrum-sensing mode, and a probability $(1-\beta)$ that a SU is engaged in data transmission. The local hypothesis test for spectrum sensing based on $\tilde{M}$ discrete-time samples at the $k^{t h}$ secondary user is

$$
\begin{array}{ccc}
\mathcal{H}_{0}: & y_{k}[n]=z_{k}[n] & n=0,1, \ldots, \tilde{M}-1 \\
\mathcal{H}_{1}: & y_{k}[n]=\mathbf{h}_{k} \mathbf{w} s_{0}[n]+z_{k}[n] & n=0,1, \ldots, \tilde{M}-1
\end{array}
$$

where the channel $\mathbf{h}_{k}$ is assumed to be constant over the $\tilde{M}$ samples. The $\tilde{M}$ complex samples are composed of $M \triangleq 2 \tilde{M}$ independent real and imaginary components [7].

The test statistic for the Neyman-Pearson-optimal energy detector is given by

$$
T_{k}=\sum_{n=0}^{\tilde{M}-1}\left|y_{k}[n]\right|^{2}=\mathbf{y}_{k}^{H} \mathbf{y}_{k}
$$

and the normalized test statistic has a central chi-square distribution with $M$ degrees of freedom under both hypotheses, where $M$ is even. Therefore, the false alarm probability follows from the chi-square cdf as [4]

$$
P_{F A, k}=e^{-\frac{\lambda_{k}}{\sigma_{k}^{2}}} \sum_{r=0}^{M / 2-1} \frac{1}{r !}\left(\frac{\lambda_{k}}{\sigma_{k}^{2}}\right)^{r},
$$

where the detection threshold $\lambda_{k}$ is a function of the target $P_{F A, k}$. Similarly, the detection probability is given by

$$
P_{D, k}=e^{-\frac{\lambda_{k}}{\left(\sigma_{s}^{2}\left|\mathbf{h}_{k} \mathbf{w}\right|^{2}+\sigma_{k}^{2}\right)}} \sum_{r=0}^{M / 2-1} \frac{1}{r !}\left(\frac{\lambda_{k}}{\left(\sigma_{s}^{2}\left|\mathbf{h}_{k} \mathbf{w}\right|^{2}+\sigma_{k}^{2}\right)}\right)^{r} .
$$

\section{Primary Receiver Performance}

From the perspective of the primary link, a missed detection at any of the SUs when the PT is active leads to interference at the primary receiver. As before, let $P_{D, k}$ represent the detection probability of secondary user $k$. Dropping the time index for brevity, write the signal at the primary receiver as

$$
y_{0}=\mathbf{h}_{0} \mathbf{x}+\sum_{k=1}^{K} F_{k} g_{k} s_{k}+z_{0}
$$

where SUs with a missed detection transmit inadvertently with power $p_{j}$ and complex channel coefficient $g_{j} \sim \mathbb{C N}\left(0, \sigma_{g, j}^{2}\right)$ to the PR, and we define the random-valued indicator function $F_{k}$ as

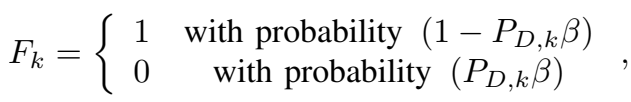

where $P_{D_{k}} \beta$ is the probability that SU $k$ is in sensing mode and has detected the presence of the PT, and thus is not producing interference. We are interested in the characteristics of the aggregate SU interference power

$$
I_{0}(\mathbf{w})=\sum_{k=1}^{K} F_{k}\left|g_{k}\right|^{2} p_{k} .
$$

Note that (9) depends on $\mathbf{w}$ through the variable $F_{k}$, since the ability of the SU to detect the PT depends on $\mathbf{w}$. The distribution of the aggregate interference in interweave networks has been approximated using tools from stochastic geometry in [5] for Poisson point process-distributed SU locations, but this is different from our system model.

Taking the expectation of the SU interference power in (9) with respect to $\left\{F_{k}\right\}_{k=1}^{K}$ and the SU-PR channels $\left\{g_{k}\right\}_{k=1}^{K}$ yields

$$
I_{0}^{\prime}(\mathbf{w})=\sum_{k=1}^{K}\left(1-P_{D, k} \beta\right) \sigma_{g, k}^{2} p_{k},
$$

where $\sigma_{g, k}^{2}$ denotes the variance of the $k$ th SU-PT channel. The PR SINR that can be computed at the PT with partial CSI is thus

$$
\gamma_{0}^{\prime}=\frac{\left|\mathbf{h}_{0} \mathbf{w}\right|^{2}}{\sigma_{0}^{2}+I_{0}^{\prime}(\mathbf{w})}
$$

where the aggregate interference $I_{0}^{\prime}(\mathbf{w})$ is a function of $\mathrm{SU}$ parameters $\beta,\left\{\sigma_{g, k}^{2}\right\}_{k=1}^{K},\left\{p_{k}\right\}_{k=1}^{K}$, and the PT beamformer w via $P_{D, k}$. 
Having defined the impact of missed detections by the SUs on the performance of the PR, we see that it is in the PT's interest to ensure that the probability of missed detection at the SUs is made as small as possible, or equivalently, that the probability of detection is made as large as possible. To this end, we introduce the paradigm of prescient beamforming in the next section in order to improve the reliability of the primary link.

\section{Prescient Primary BeAmforming}

\section{A. Direct PR SINR maximization}

A first approach to constructing a prescient transmission scheme would be for the PT to directly optimize the PR SINR $\gamma_{0}^{\prime}$ under the transmit power constraint:

$$
\begin{aligned}
& \max _{\mathbf{w}} \frac{\left|\mathbf{h}_{0} \mathbf{w}\right|^{2}}{\sigma_{0}^{2}+I_{0}^{\prime}(\mathbf{w})} \\
& \text { s.t. } \mathbf{w}^{H} \mathbf{w} \leq P / \sigma_{s}^{2} .
\end{aligned}
$$

A similar problem can be posed in the case of maximizing the average PR SINR $E_{\mathbf{h}_{0}, \ldots, \mathbf{h}_{K}}\left\{\gamma_{0}^{\prime}\right\}$. Note that the interference term in the PR SINR is a function of the transmit signal itself. While signal-dependent interference is a well-studied problem in radar signal processing [6], in our case this dependence manifests itself in a much more complicated and non-linear fashion involving exponential terms (and special functions in the case of $\bar{\gamma}_{0}$ ). An analytical optimization of (12) over w appears to be intractable, but one could attempt to find the optimal beamformer using a gradient descent algorithm, recognizing of course that global optimality is not guaranteed due to the non-convexity of the objective function.

The Karush-Kuhn-Tucker (KKT) conditions for a stationary point of (12) can be computed as

$$
\begin{aligned}
& \nabla_{\mathbf{w}}\left(\gamma_{0}^{\prime}\right)+\mu \mathbf{w}=\mathbf{0} \\
& \mathbf{w}^{H} \mathbf{w}-P / \sigma_{s}^{2} \leq 0 \\
& \mu\left(\mathbf{w}^{H} \mathbf{w}-P / \sigma_{s}^{2}\right)=0, \quad \mu \geq 0,
\end{aligned}
$$

where the gradient of the PR SINR is given by

$$
\begin{aligned}
\nabla_{\mathbf{w}}\left(\gamma_{0}^{\prime}\right)= & \frac{\left(\mathbf{h}_{0}^{H} \mathbf{h}_{0}\right) \mathbf{w}}{\sigma_{0}^{2}+I_{0}^{\prime}(\mathbf{w})} \\
& +\frac{\beta\left|\mathbf{h}_{0} \mathbf{w}\right|^{2} \sigma_{s}^{2} \sum_{k=1}^{K}\left[\sigma_{g, k}^{2} p_{k}\left(A_{k}-B_{k}\right)\right]}{\left(\sigma_{0}^{2}+I_{0}^{\prime}(\mathbf{w})\right)^{2}}(16) \\
\Omega_{k}(\mathbf{w})= & \frac{\lambda_{k} / 2}{\sigma_{s}^{2}\left|\mathbf{h}_{k} \mathbf{w}\right|^{2}+\sigma_{k}^{2}} \\
A_{k}= & \Omega_{k}(\mathbf{w}) e^{-\Omega_{k}(\mathbf{w}) \mathbf{h}_{k}^{H} \mathbf{h}_{k} \mathbf{w}} \\
& \times \sum_{r=0}^{M / 2-1}\left\{(r !)^{-1}\left(\Omega_{k}(\mathbf{w})\right)^{r}\right\} \\
B_{k}= & e^{-\Omega_{k}(\mathbf{w})} \frac{\mathbf{h}_{k}^{H} \mathbf{h}_{k} \mathbf{w}}{\sigma_{s}^{2}\left|\mathbf{h}_{k} \mathbf{w}\right|^{2}+\sigma_{k}^{2}} \\
& \times \sum_{r=0}^{M / 2-1}\left\{(r-1 !)^{-1}\left(\Omega_{k}(\mathbf{w})\right)^{r}\right\}
\end{aligned}
$$

However, since the problem is non-convex the KKT conditions are merely necessary and not sufficient for optimality.

\section{B. Combined MRT and Multicast Beamforming}

While the gradient search algorithm described above returns at least a locally optimal prescient beamformer, it is desirable to investigate designs based on simpler optimization procedures. To this end, consider the following two extreme cases for the choice of $\mathbf{w}$ :

- Disregard SUs, focus only on PR: If the PT disregards the presence of the SUs and focuses only on maximizing the signal strength at the PR, the optimal choice for $\mathbf{w}$ is the maximum-ratio transmit beamformer:

$$
\mathbf{w}_{M R T}=\frac{\sqrt{P} \mathbf{h}_{0}^{H}}{\sigma_{s}\left\|\mathbf{h}_{0}\right\|} .
$$

- Disregard PR, focus only on SUs: At this extreme, the PT ignores the PR and focuses only on improving the signal strength at the SUs (particularly those that could produce the most interference at the PR). This is similar to a multicast (MC) downlink scenario, where priority is given to certain key users. A reasonable choice for the transmit beamformer in this case would maximize the weighted average of the SNRs at the SUs:

$$
\mathbf{w}_{M C}=\arg \max _{\mathbf{w}} \sum_{k=1}^{K} p_{k} \sigma_{g, k}^{2}\left|\mathbf{h}_{k} \mathbf{w}\right|^{2},
$$

where the weight $p_{k} \sigma_{g, k}^{2}$ measures the interference impact of the $k$ th $\mathrm{SU}$ at the PR. It is easy to see that the solution to (21) is given by the dominant singular vector of $\mathbf{H}_{S}^{H} \boldsymbol{\Sigma}_{g} \mathbf{H}_{S}^{H}$ scaled by $\sqrt{P / \sigma_{s}^{2}}$, where $\mathbf{H}_{S}=$ $\left[\begin{array}{lll}\mathbf{h}_{1}^{T} & \ldots & \mathbf{h}_{K}^{T}\end{array}\right]^{T}$ and $\boldsymbol{\Sigma}_{g}$ is a diagonal matrix with entries $p_{k} \sigma_{g, k}^{2}, k=1, \cdots, K$.

Given that the prescient beamforming objective is to balance these two competing goals, a sensible and mathematically tractable approach would be to choose $\mathbf{w}$ as some linear combination of the solutions:

$$
\mathbf{w}=\alpha \mathbf{w}_{M R T}+(1-\alpha) \mathbf{w}_{M C} \quad 0 \leq \alpha \leq 1,
$$

where the optimal value of $\alpha \in[0,1]$ can be found by a simple line search. We will see later in the simulations that this approach performs similarly to the gradient search for maximizing the PR SINR.

To conclude this section, we remark that the essence of the prescient beamforming schemes is to boost the received SNRs at the SUs while simultaneously maintaining a reliable link to the PR. Naturally, increasing the SNR at the SUs will also improve the overall detection probability in a cooperative sensing system where a global decision is made at a fusion center [7], [8]. Therefore, the prescient beamforming techniques proposed thus far assuming non-cooperative spectrum sensing can be applied directly without change to a cooperative sensing scenario. 


\section{Simulation Results}

In this section, we present the results of several numerical experiments to verify the improvement in primary link performance with prescient beamforming. All results in this section are based on the partial CSI model with instantaneous CSI of the PT-PR and PT-SU links, and only statistical CSI of the SU-PR links available to the primary transmitter. Each channel realization for all terminals is drawn from a zero-mean unitvariance circularly symmetric complex Gaussian distribution, and all results are averaged over 1000 channel realizations. The AWGN variance at all receivers is assumed to be unity, i.e., $\sigma_{k}^{2}=1 \forall k$. For every non-cooperative sensing scenario we assume a sensing probability of $\beta=0.9$, target false alarm rate $P_{F A}=10^{-3}$, and sample size of $M=4$ used by the SUs for detection. The gradient-PBF algorithms are run 5 times for each set of channel realizations with four random initializations and an initialization based on the naïve MRT precoder to reduce the likelihood of a local maximum; the best-performing precoding solution is chosen as the result.

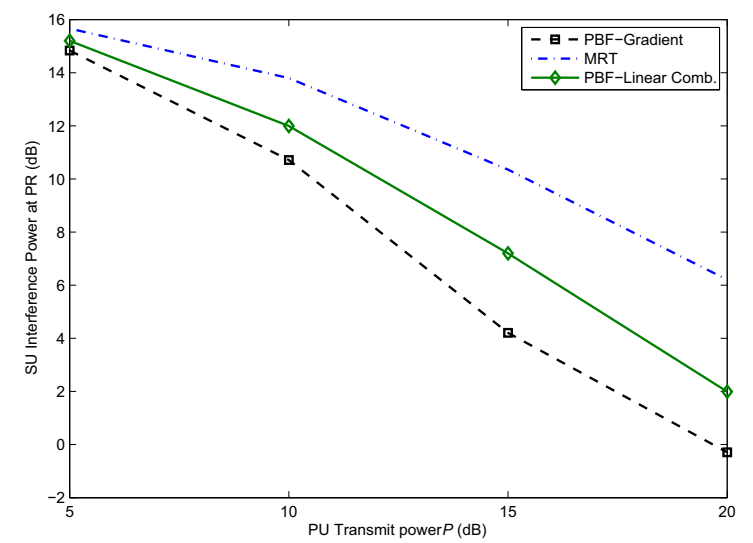

Fig. 2. SU interference power at primary receiver for PBF and MRT schemes with non-cooperative spectrum sensing, $p_{k}=20 d B, K=5, N=4$.

Fig. 2 displays the aggregate SU interference at the primary receiver for the various beamforming schemes, with the same parameters as in Fig. 3. We observe that the gradient-PBF approach virtually eliminates $\mathrm{SU}$ interference due to missed detections at high SNRs.

In Fig. 3, we compare the primary SINR for prescient versus naïve transmission as a function of the primary transmit power. Here, the fixed parameters are $N=4, K=5, p_{k}=20 \mathrm{~dB}$. The exploitation of SU CSI affords a performance increase of around $3 d B$ at low to moderate SNRs. The combined MRTMC beamformer of (22), referred to as "linear combination" in the plot, has a negligible SINR loss compared to the direct approach in (12). The corresponding increase in PR SINR under the prescient schemes is not of the same magnitude as the interference mitigation in Fig. 2, since the PBF transmission also degrades the desired signal power when compared to MRT.

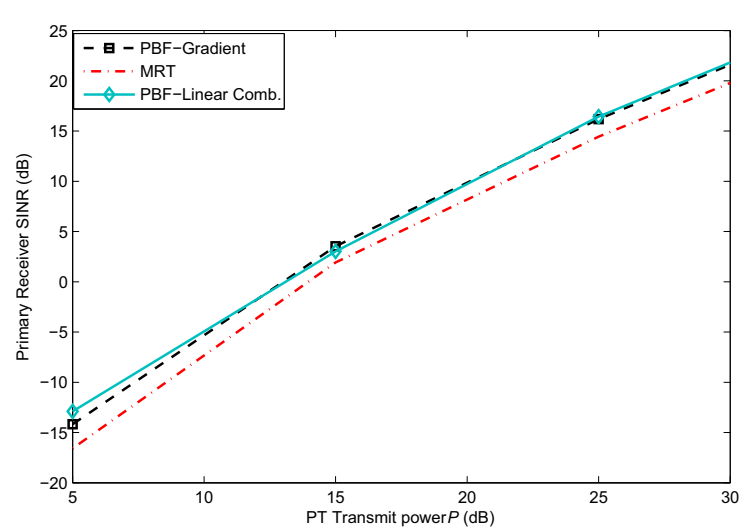

Fig. 3. Primary receiver SINR for PBF and MRT transmission. SU transmit power $p_{k}=20 d B, K=5, N=4, M=4$.

\section{CONCLUSION}

In this work we considered the novel problem of linear precoding by the primary transmitter to increase the detection probability at spectrum-sensing cognitive radios, and thereby decrease the inadvertent interference at the primary receiver due to imperfect spectrum sensing. We presented two prescient beamforming schemes with differing complexities that preemptively mitigate secondary interference for non-cooperative spectrum sensing. Numerical results demonstrate that the primary link performance is improved under the proposed prescient beamforming methods. In forthcoming work, we have investigated the design of prescient precoding techniques for interweave networks with multiple primary receivers, as well as applications to cooperative spectrum sensing scenarios.

\section{REFERENCES}

[1] A. Goldsmith, S. A. Jafar, I. Maric, and S. Srinivasa, "Breaking spectrum gridlock with cognitive radios: An information theoretic perspective,' Proc. IEEE, vol. 97, pp. 894-914, May 2009.

[2] R. Zhang, T. J. Lim, Y.-C. Liang, and Y. Zeng, "Multi-antenna based spectrum sensing for cognitive radios: A GLRT approach," IEEE Trans. Commun., vol. 58, no. 1, pp. 84-88, Jan. 2010.

[3] R. Zhang and Y. C. Liang, "Exploiting multi-antennas for opportunistic spectrum sharing in cognitive radio networks," IEEE J. Sel. Topics Signal Process., vol. 2, pp. 88-102, Feb. 2008.

[4] S. M. Kay, Fundamentals of Statistical Signal Processing Vol. II: Detection Theory. Prentice Hall, 1998.

[5] A. Ghasemi and E. S. Sousa, "Interference aggregation in spectrumsensing cognitive wireless networks," IEEE J. Sel. Topics Signal Process., vol. 2, no. 1, pp. 41-56, Feb. 2008.

[6] S. U. Pillai, H. S. Oh, D. C. Youla, and J. R. Guerci, "Optimum transmitreceiver design in the presence of signal-dependent interference and channel noise," IEEE Trans. Inf. Theory, vol. 46, no. 2, pp. 577-584, Mar. 2000.

[7] J. Ma, G. Zhao, and Y. Li, "Soft combination and detection for cooperative spectrum sensing in cognitive radio networks," IEEE Trans. Wireless Commun., vol. 7, no. 11, pp. 4502-4507, Nov. 2008.

[8] G. Xiong, S. Kishore, and A. Yener, "On performance evaluation of cooperative spectrum sensing in cognitive radio networks," in Proc. CISS, pp. 1-6, Princeton, NJ, Mar. 2010. 